\title{
Hydrostatic optimization of inkjet-printed films
}

Hongki Kang

Electrical Engineering and Computer Sciences University of California at Berkeley

Technical Report No. UCB/EECS-2010-44

http://www.eecs.berkeley.edu/Pubs/TechRpts/2010/EECS-2010-44.html

April 22, 2010 
Copyright (C 2010, by the author(s).

All rights reserved.

Permission to make digital or hard copies of all or part of this work for personal or classroom use is granted without fee provided that copies are not made or distributed for profit or commercial advantage and that copies bear this notice and the full citation on the first page. To copy otherwise, to republish, to post on servers or to redistribute to lists, requires prior specific permission. 


\title{
Hydrostatic optimization of inkjet-printed films
}

\author{
by Hongki Kang
}

\section{Research Project}

Submitted to the Department of Electrical Engineering and Computer Sciences, University of California at Berkeley, in partial satisfaction of the requirements for the degree of Master of Science, Plan II

Approval for the Report and Comprehensive Examination:

\section{Committee:}

Professor Vivek Subramanian

Research Advisor

Date:

Professor Stephen Morris

Second Reader

Date: 


\section{Table of Contents}

Table of Contents ............................................................................... 2

I. Introduction ........................................................................................ 3

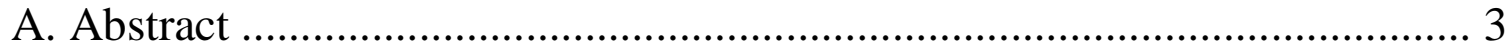

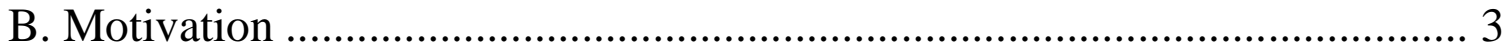

C. Contact Angle Hysteresis and Its Measurement ...................................... 5

II. Experimental Setup, Results and Qualitative Analysis................................ 8

A. Experimental Setup ............................................................................. 8

B. Experimental Results and Qualitative Analysis .................................... 11

a. Experimental Results ...................................................................... 11

b. Qualitative Analysis ...................................................... 14

III. Quantitative Analysis based on 1D Hydrostatic Model ............................... 17

A. Inkjet-Printed Film Thickness Model ................................................ 17

B. Bulging of Printed Film beyond Advancing Contact Angle ..................... 19

a. Two Lines Impinging ................................................................ 20

b. Generalization to Many Printed Lines ............................................. 22

C. Separation of Printed Film below Receding Contact Angle ................... 25

a. Film Separation Model .............................................................. 25

b. Multiple Nozzle Printing ........................................................... 28

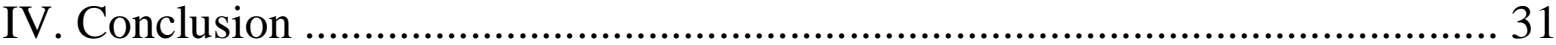

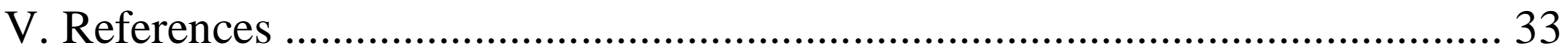

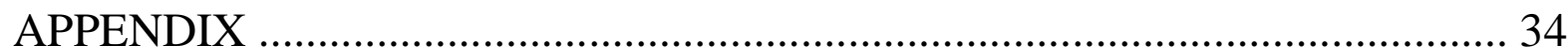




\section{Introduction}

\section{A. Abstract}

In this report, we study the optimization of the geometry of inkjet-printed polymer films and develop a simple analytic framework to understand our results and establish limitations on inkjet-printed patterns. We show how drop spacing and ink concentration affect the thickness of a printed film and how hydrostatic conditions with contact angle hysteresis have to be considered to print optimized rectangular features. If advancing and receding contact angle are not taken into account, printed features will either bulge or breakup into smaller beads. Thus, we provide a comprehensive analysis of the limits of film formation using regular assemblies of droplets.

\section{B. Motivation}

Printing technologies have emerged as a promising technique to realize low-cost integrated circuits. Among several printing options, drop-on-demand inkjet printing is an attractive tool for low-cost electronics fabrication due to its precise, flexible control and cost-efficient additive processing. Inkjet printing can generate patterns of metals, semiconductors or insulators by jetting ink drops of the appropriate materials on a substrate at desired locations. Dots form a line, and lines form a film by merging.

Printed films play a significant role in electronic devices such as transistors, passive circuit elements, displays, and solar cells. Various aspects of these electrically active printed films need to be understood and controlled in these electronic applications. For example, the thickness of the insulating layer of a transistor or a capacitor has to be controlled for a precise specific capacitance and to avoid leakage. Similarly, for printed light emitting diodes and solar cells, 
thickness control is required for light emission efficiency and absorption. The surface of printed films needs to be smooth for better field endurance in capacitors and for less carrier scattering in active devices. Its overall geometry must be controlled both for electrical performance and for efficient circuit layout.

In order to make inkjet printing more controllable and technologically feasible, it is important to understand the nature of printed features and generate design rules for inkjet printing [1]-[2]. An understanding of the various rheological effects present during printing of films is necessary for the realization of optimized, reproducible inkjet-printed devices. Recently, various authors have demonstrated an understanding of drying processes based on the evaporation parameters of inks, causing various surface topographies of printed films to manifest themselves [3]-[5]. To solve some of the observed effects, Tekin et. al. used a multiple-pass inkjet-printing technique to improve homogeneity of a film [6]. The multiple-pass technique, called multilevel matrix in their work, prints matrixes which consist of independent drops and requires back and forth movement of nozzles over the film. They thus offer an experimentally derived solution to film formation challenges in droplet-based printing. Unfortunately, this technique is problematic from a manufacturing perspective, since the multiple passes dramatically reduce throughput and deleteriously impact process cost. What is desired is to obtain a quantifiable understanding of the hydrostatic effects impacting pattern shape control, and to use this understanding to optimize printing conditions to realize well-controlled shapes. In this work, we examine the formation of contiguous film from droplets printed in a unitary raster scan, which is more appropriate for faster single-pass printing. We develop a simple analytical framework to establish the limitations imposed by hydrostatic considerations with contact angle hysteresis on pattern formation using the same, thus providing optimization guidelines for formation of films using 
inkjet printing. This is particularly important, since it established design and printing relationships that determine the limits over which well-defined structures can be printed.

This report is organized as follows - we begin by establishing the basic measurement techniques used in this work, and then establish the proposed theoretical framework. Finally, we examine the implications of the same. The concept of contact angle hysteresis and its general measurement technique will be briefly discussed in the next section. In the body of this report, experimentally observed phenomena related to firm formation are presented, and these are used to develop a quantitative model for film formation. Finally, the results will be analyzed both qualitatively and quantitatively to form an understanding of the implications of contact angle hysteresis on film formation.

\section{Contact Angle Hysteresis and Its Measurement}

As well known, a sessile drop on a substrate has a contact angle defined by the Law of YoungDupré. The surface tensions between gas/liquid, liquid/solid and solid/gas are equilibrated by forming the equilibrium contact angle. When the substrate gets dirty either chemically (defects) or physically (roughened), the contact angle does not only have one value. If there are defects which attract the liquid, more energy is needed to break up the interaction between the defects and the drop. Also, when the surface is rough, the macroscopic contact angle can have a range of values because the rough surface has non-zero angle slope which changes the macroscopic angle. When the volume of a liquid drop is increased, the angle beyond which the drop starts expanding is defined as 'advancing contact angle'. Also, while the volume of a liquid drop is decreased, the angle below which the drop retreats is 'receding contact angle'. In the case of dirty surface, the 
advancing contact angle and receding contact angle are not identical, resulting in nonzero contact angle hysteresis.

Contact angle hysteresis can be measured based on the definitions of the various contact angles. With a supply of ink into a drop to wet the substrate, measured contact angle when the drop just starts advancing can be determined as the advancing contact angle. Similarly, by sucking the ink through a needle to recede the drop, we can measure the retreating contact angle as shown in Figure 1(a). In order to minimize the effect of surface tension between the needle and the liquid, it is desirable to use a needle with low surface energy. Instead of controlling the volume of a drop, a tilted substrate which makes a droplet advance and recede by gravity can be used to measure the hysteresis as shown in Figure 1(b).
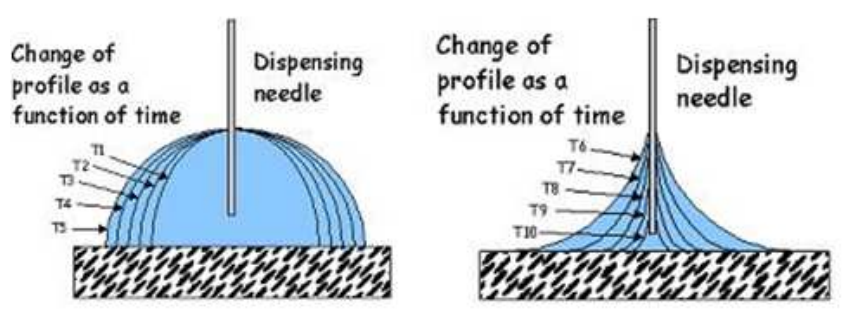

(a)

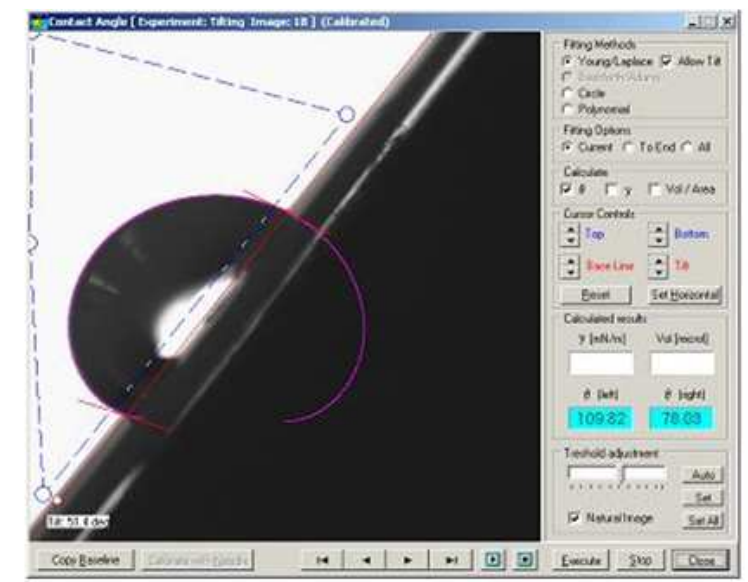

(b)

Figure 1. Methodologies to measure dynamic contact angles (source: KSV Instruments Ltd., http://www.ksvltd.com/content/index/keydca) (a) using needle to advance/recede the drop on a flat substrate. (b) Tilting the substrate to advance/recede the drop.

In this work, the contact angle hysteresis plays the most important role in explaining what we observe and anticipating the pattern shapes. Thus, it will be repeatedly mentioned through the 
report. Also, the former measurement method of using a needle is used to measure the contact angles in our experiments. Given the explanation of contact angle hysteresis above, we are now ready to proceed with experiments and analyses of the implications of this phenomenon on film formation. 


\section{Experimental Setup, Results and Qualitative Analysis}

\section{A. Experimental Setup}

To facilitate the development and verification of a model of the hydrostatic considerations in film formation, we study film formation experimentally using droplet-on-demand inkjet printing. Our experiment is conducted with two drop-on demand inkjet printers, a custom-built system based on a Microfab piezoelectric jetting head and the Dimatix Materials Printer DMP-2800. The Microfab system jets $100 \mathrm{pL}$ drops through a single $60 \mu \mathrm{m}$ orifice at a frequency of about 25 Hz. The Dimatix system jets $10 \mathrm{pL}$ drops through up to 16 nozzles at $2 \mathrm{kHz}$.

For this work, we print a polymer ink, poly(4-vinylphenol), PVP, dissolved in 1-hexanol. PVP is commonly used dielectric material in organic TFTs, with suitable cross-linking [7]-[8]. PVP ink usually contains a crosslinker material, but the crosslinker is not included in this experiment to ensure a simpler solvent system. Concentrations of PVP inks used in this experiment are 7.60 $\mathrm{wt} \%$ and $14.13 \mathrm{wt} \%$. While our experimental data and verification is limited to this important material system, the methodology and modeling developed herein is more generally application, provided appropriate parameters are used for different ink systems.

To ensure highly controlled substrate conditions requisite for good modeling, we print on two different glass substrates, Fisher brand No. 12-550C microscope slide (microscope slide) and Corning 1737 (Corning glass) from Corning Inc, a display grade alkaline earth boroaluminosilicate glass. The glass substrates are cleaned by sequential sonication in soap water, acetone, and isopropyl alcohol for 20 minutes each. 
The $7.60 \mathrm{wt} \%$ PVP ink on the two glass substrates shows a similar advancing contact angle, but different retreating contact angles, as measured by a Kruss contact angle measurement system. The advancing contact angle for the microscope slide is $27.2 \pm 1.5^{\circ}$, and the receding contact angle is close to zero. The advancing contact angle for Corning glass is $26.0 \pm 1.5^{\circ}$ and receding contact angle is $14.4 \pm 0.6^{\circ}$. The advancing and receding contact angles are dynamic contact angle since related to the flow of fluid. The measured diameter of a jetted single drop is about $115 \mu \mathrm{m}$ for a $100 \mathrm{pL}$ sessile drop and about $45 \mu \mathrm{m}$ for a $10 \mathrm{pL}$ sessile drop on both substrates.

Printed films, exclusively rectangles in this work, are built up in a raster-scan method with a constant drop spacing. This drop spacing is the same in the slow-print (left-to-right) and fastprint (bottom-to-top) directions as shown in Figure 2. The PVP films are printed and dried on the printing platen at $30^{\circ} \mathrm{C}$. After drying, PVP films are characterized with an optical microscope and a mechanical stylus profilometer.

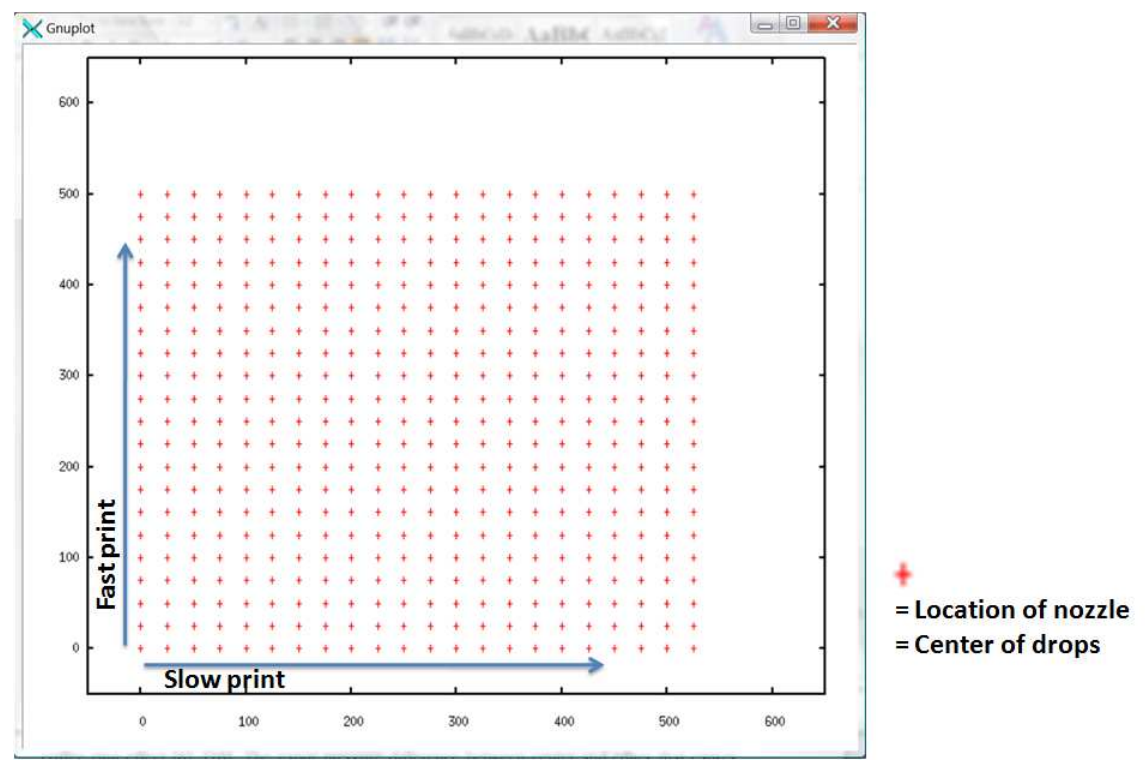

Figure 2 A map for inkjet printing drops to form $500 \mu \mathrm{m}$ square. The red cross mark is where the inkjet nozzle is moved to and jets drops. The jetted drop lands on the spot and spreads to meet equilibrium condition. 
Every printed square film in this work shows significant coffee-ring effect around its boundary as shown in Figure 3. As explained by Deegan et. al., this "coffee ring" is formed due to outward capillary flow caused by pinned contact lines and enhanced evaporate at the edge of a partially wetting film [9]. It has been demonstrated that dual solvent systems with different vapor pressure can suppress the coffee-ring effect [6], [10]. The vapor pressure difference between center and edges that causes the outward capillary flow is reduced because the concentration of lower vapor pressure solvent is higher at the edges due to faster evaporation of the solvent with higher vapor pressure. However, the dual-solvent system is not used in this work in order to develop a hydrostatic model based on a single, time-invariant contact angle. Excluding the coffee-ring edges, the thickness of the films is almost constant since the evaporation rate near the center is constant as shown in Figure 3(b). Additionally, we note that the central flat region of dielectric films can be used in organic TFTs despite a coffee ring edge; indeed this structure is advantageous since the central region is extremely flat, unlike the dome-shaped profile commonly observed in films formed using coffee-ring retarding concepts [11]. The average thickness of the central flat region is defined as the thickness of the polymer film in this work. Since the modeling of hydrostatic effects considered in this work is important for timescales before the films dry, the presence of coffee-rings does not influence modeling or verification efforts, nor does it affect the predictive capability of the model. Indeed, the model can be extended to account for multi-solvent systems by introducing time-variant contact angles, but this will not be done here. 


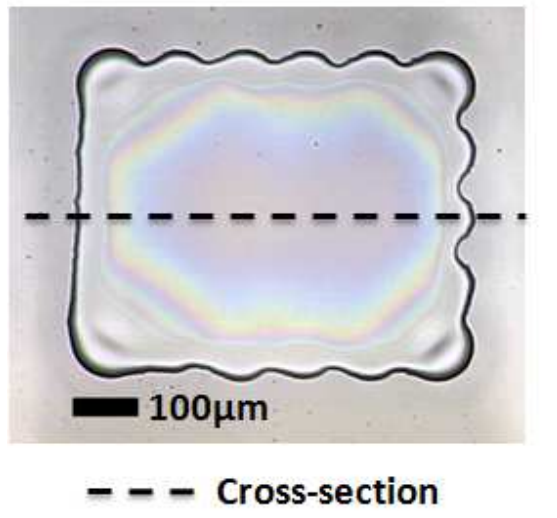

(a)

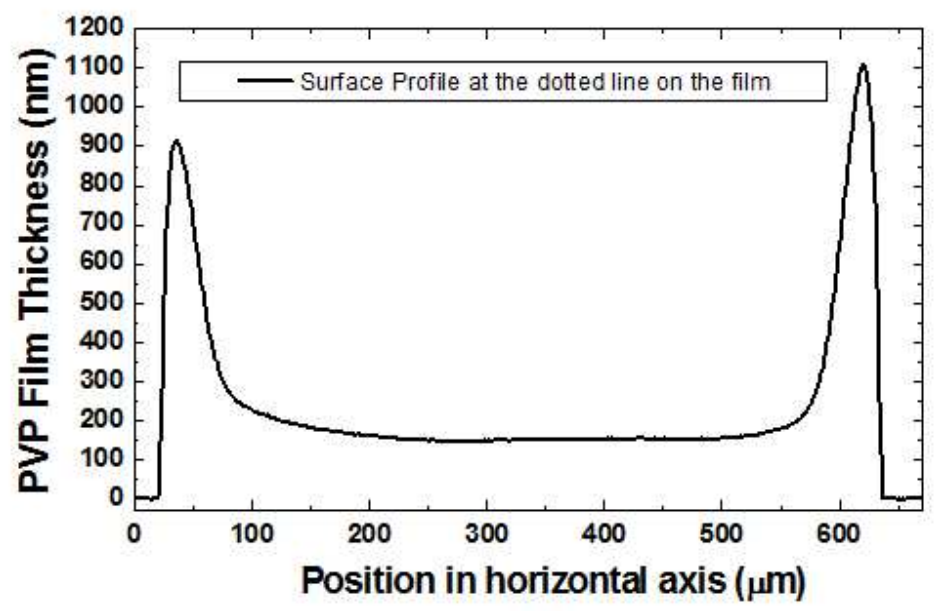

(b)

Figure 3. (a) Microscope image of a $500 \mu \mathrm{m}$ square $7.60 \mathrm{wt} \%$ PVP film on a microscope slide, which is printed by the custom-built inkjet printer at $100 \mu \mathrm{m}$ drop spacing (b) Surface profile of this film's cross-section.

\section{B. Experimental Results and Qualitative Analysis}

\section{a. Experimental Results}

To collect the requisite data for material development and verification, we investigate how the thickness and pattern fidelity of printed films varies as drop spacing changes. As shown in Figure 4, the measured thicknesses of $500 \mu \mathrm{m}$ square films printed by the Microfab system and those of $200 \mu \mathrm{m}$ square films printed by Dimatix decrease monotonically as drop spacing increases. The minimum thickness in a given ink-substrate system is determined by the spacing required for drop overlap. As shown in Figure 5, drops do not merge together and the film is separated when drop spacing is larger than the diameter of a single drop. Also the ink concentration affects the thickness of printed films. Doubling the amount of PVP (from $7.6 \mathrm{wt} \%$ 
to $14.13 \mathrm{wt} \%$ ) yields doubled film thickness. We see the same tendency of monotonically decreasing thickness with increasing drop spacing with the higher concentration ink.

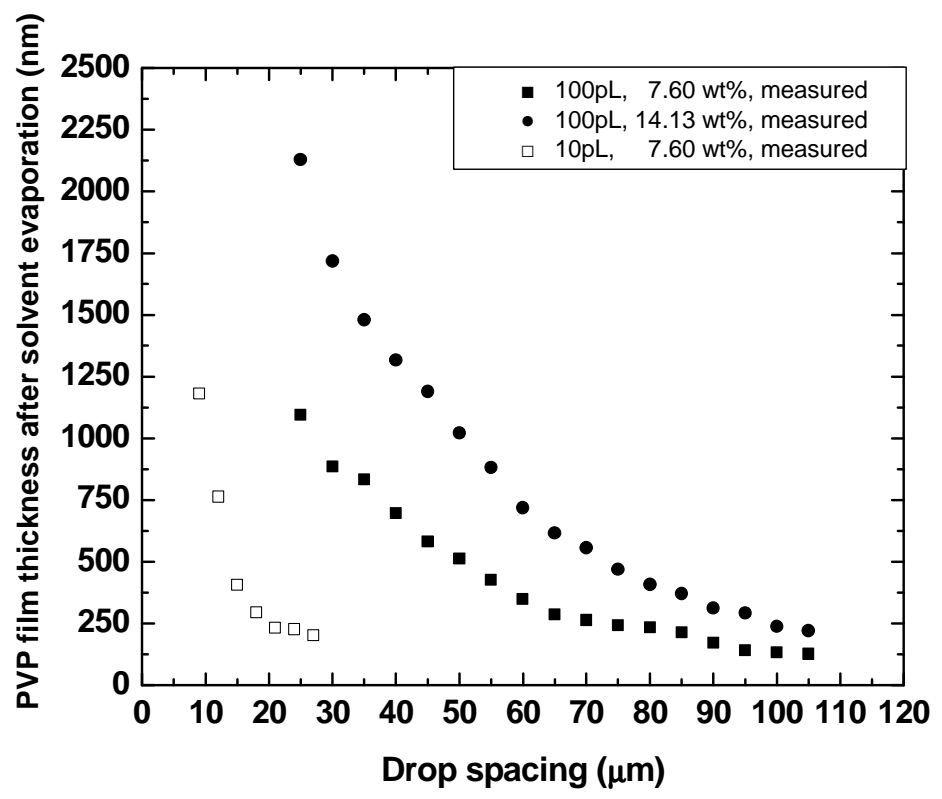

Figure 4. Film thickness modeling of equation (1) with extended boundary (See supporting information for more detail) and measurement data for $500 \mu \mathrm{m}$ square PVP films printed by the Microfab printer $(115.5 \mu \mathrm{m}$ diameter of a circular cap on Corning 1737) and $200 \mu \mathrm{m}$ by the Dimatix printer $(45 \mu \mathrm{m}$ diameter).

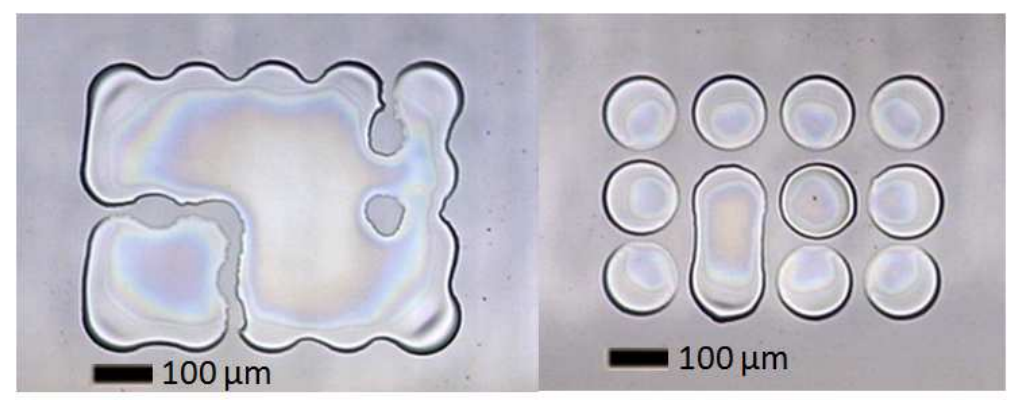

(a)

(b)

Figure 5. Microscope image of $500 \mu \mathrm{m}$ square $7.60 \mathrm{wt} \%$ PVP films on Corning 1737; drop spacing/diameter of a single drop (a) 1.04, (b) 1.21. 
In addition to the film thickness, the shape of the formed films also varies as the drop spacing changes. As drop spacing decreases, the square film starts bulging at the left edge and eventually bulges on every edge at sufficiently low spacing, forming a circular shape as shown in Figure 6.

We observe another interesting phenomenon when we print larger films. The film may separate into smaller beads as we try to print larger film. This results in the pattern breakup shown in Figure 7. The top-right corner drops, which are jetted last, are separated from the film with $40 \mu \mathrm{m}$ drop spacing. Larger drop spacing results in more severe film breakup.

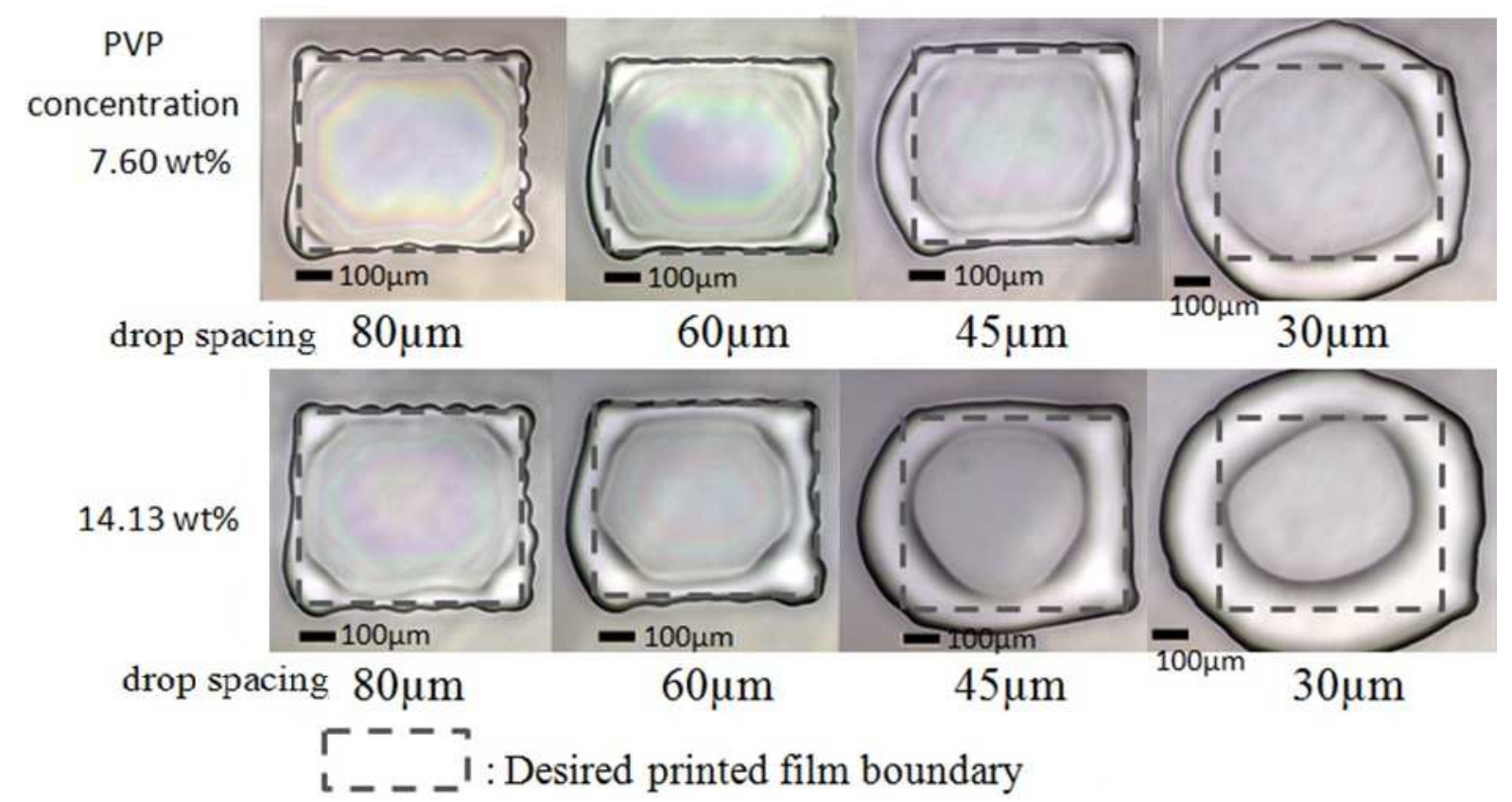

Figure 6. Microscope images of $500 \mu \mathrm{m}$ square $7.60 \mathrm{wt} \%$ PVP films on Corning 1737 printed with labeled ink, at labeled drop spacing; 


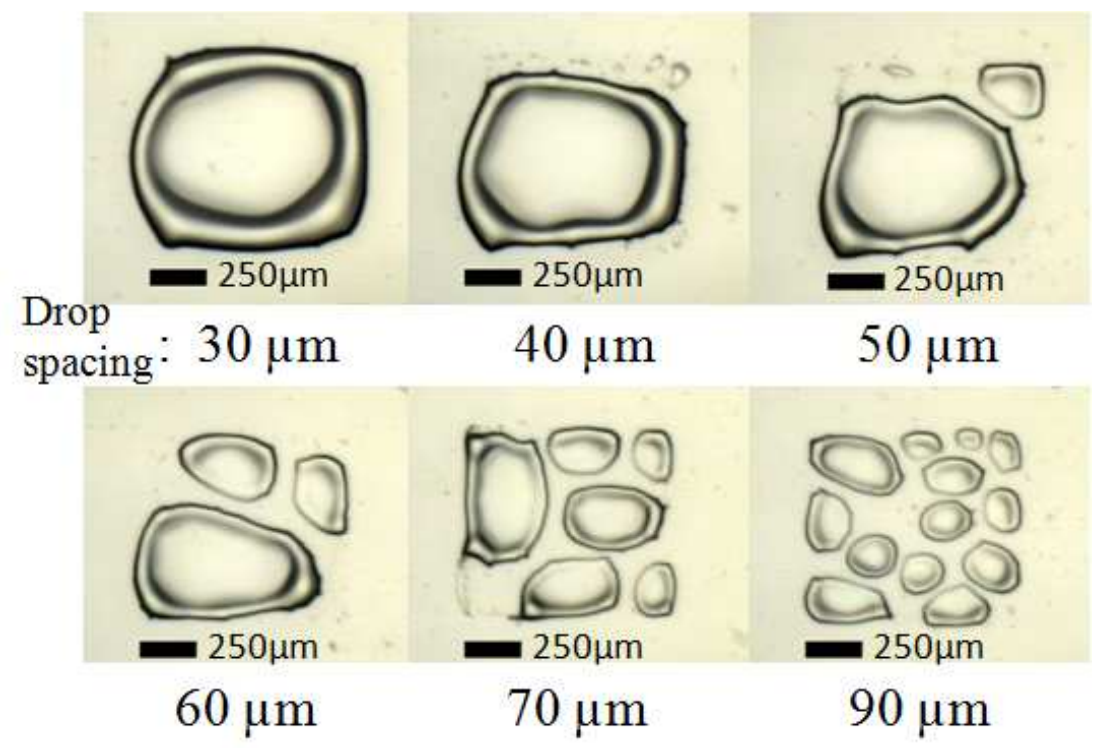

Figure 7. Film separation of $1 \mathrm{~mm}$ square $7.60 \mathrm{wt} \%$ PVP film on Corning 1737 as drop spacing increases.

\section{b. Qualitative Analysis}

In a uniform film, assumed to be much thinner than the upper limit on film thickness imposed by gravity (see Appendix), the final PVP film thickness is determined by the total volume of PVP ink per unit area. Assuming constant drop spacing in both print directions, the number of jetted drops in printed films of the same size is inversely proportional to this constant drop spacing value. Therefore, the thickness of the film decreases monotonically as drop spacing increases.

If the volume of ink in a film is too much such that contact angle at the edges of the film is higher than the requisite advancing contact angle, then the boundary of the film expands as the excess ink flows outward to form an appropriate advancing contact angle at the edges for surface energy minimization. Since we raster-scan the film from left to right, the contact angle at each 
edge of the printed bead decreases as more lines are added to the right. Therefore, in Figure 6, bulging tends to occur on the left hand side when the contact angle is initially higher than advancing contact angle. As the contact angle fall beneath the advancing contact angle, the bulging does not occur any more, resulting no bulging on the right hand side. When more lines are added with relatively large drop spacing, the contact angle continues to decrease and fall beneath the receding contact angle. It then causes the right edge to be pulled to the left, resulting in scalloped morphology.

In order to understand the film breakup, we also need to consider the retreating contact angle. As the wetted footprint of a pattern is expanded through further printing, the growing bead's contact angle may fall due to geometric considerations depending on the volume of ink deposited per increase in substrate area covered. Should a growing bead's contact angle fall beneath the receding contact angle as printing proceeds, the contact line will retreat. Thus, the film breakup occurs as we print larger films.

If the receding contact angle is near zero which means almost total pinning, then the film breakup does not happen because the bead's contact angle is always higher than the receding contact angle. In case of printed features on the microscope slide whose retreating contact angle is near zero, $2 \mathrm{~mm}$ square films are able to be printed with almost no breakup as shown in Figure 8. Although the film with $100 \mu \mathrm{m}$ spacing shows small inside breakups, the films maintain their square boundary very well. As shown in Figure 9, much larger films including $5 \mathrm{~mm}$ square film can also be printed on the same substrate, which is impossible on the Corning glass. 


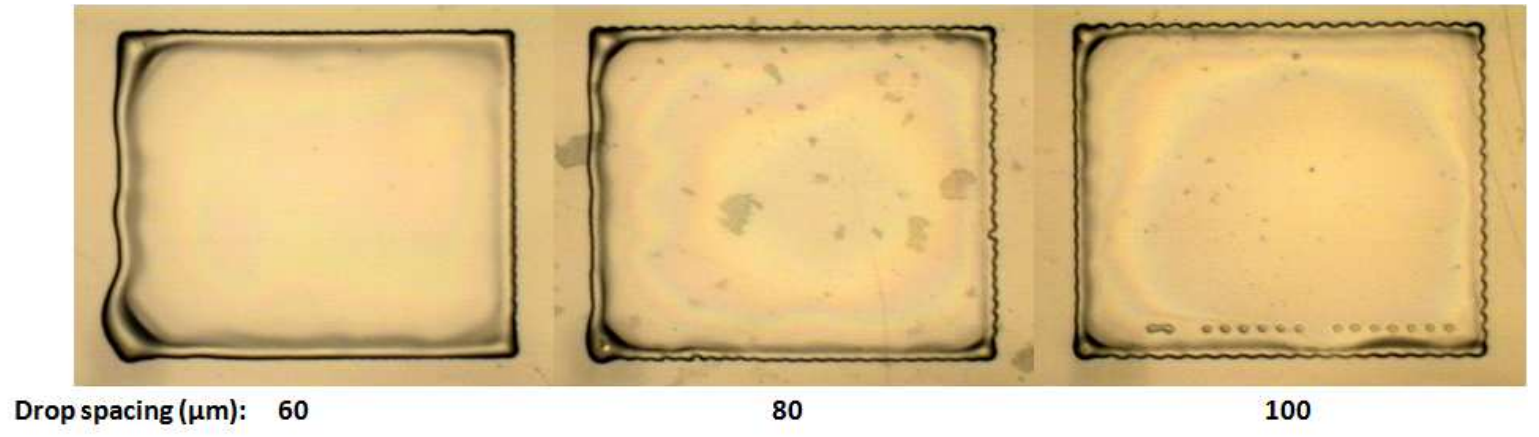

Figure $82 \mathrm{~mm}$ square films of $7.60 \mathrm{wt} \%$ PVP film on the microscope slide with different drop spacing.

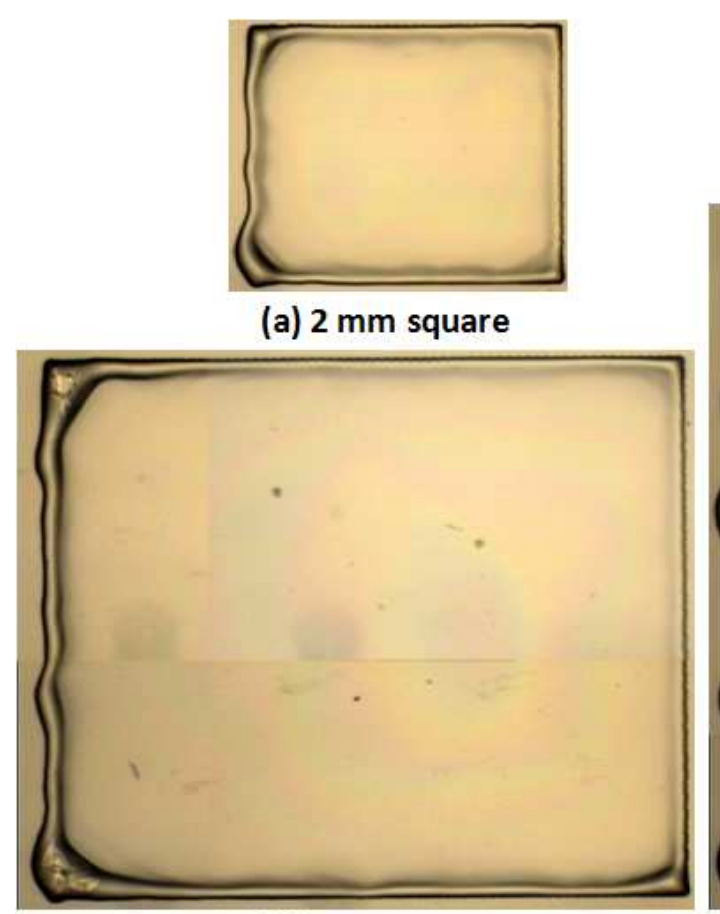

(b) $4 \mathrm{~mm}$ square

\section{Drop spacing: $60 \mu \mathrm{m}$}

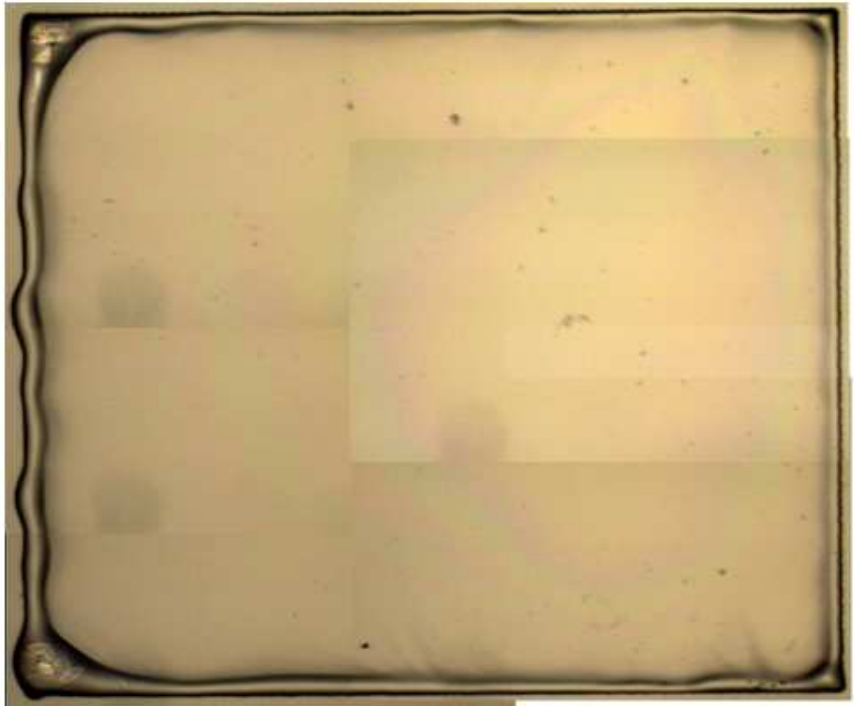

(c) $5 \mathrm{~mm}$ square

Figure 9 Square films of $7.60 \mathrm{wt} \%$ PVP on the microscope slide with $60 \mu \mathrm{m}$ drop spacing; size of (a) $2 \mathrm{~mm}$, (b) $4 \mathrm{~mm}$ and (c) $5 \mathrm{~mm}$. 


\section{Quantitative Analysis based on 1D Hydrostatic Model}

To proceed with development of analytical models for film formation, we examine film thickness limitations as well as pattern fidelity limitations as a function of hydrostatic considerations.

\section{A. Inkjet-Printed Film Thickness Model}

As discussed in the previous section, PVP film thickness is determined by the total volume of PVP ink per unit area. Assuming that a printed film is large compared to the size of a single drop, the final thickness of the dried printed film, $h$, is proportional to the drying ratio and the total volume of the film and inversely proportional to the area of the film:

$$
h=C \times \frac{n^{2} V_{d r o p}}{(n d)^{2}}=C \frac{V_{d r o p}}{d^{2}}
$$

where the volume of a single drop is $V_{d r o p}$, the drop spacing is $d, C$ is experimentally extracted drying ratio, and the number of lines forming the square is $n$. However, if the size of a wetting drop approaches the size of the printed film, the size of the drop has to be included in the area of the film. If the center of drop is jetted onto the square of designed size, then the total area of printed square includes the extended boundary of jetted drop radius, $2 R$ on both sides. It is significant when the radius is comparable to the size of the film. Assuming two sides of a rectangle film shown in Figure $\mathbf{1 0}$ are a and $\mathrm{b}$, the radius of a single drop is $R$, the volume of a single drop is $V_{\text {drop }}$, the drop spacing is $d$ and $C$ is experimentally extracted drying ratio, the thickness of the dried printed film, $h$, can be derived in the following way. 


$$
\begin{aligned}
& h=\frac{(\text { drying ratio }) \times(\text { total volume of a film })}{(\text { total area of a film with extended boundary })} \\
& =C \times\left\{\left(\frac{a}{d}+1\right) \times\left(\frac{b}{d}+1\right) \times V_{d r o p}\right\} \div\{(a+2 R) \times(b+2 R)\} \\
& =C \times\left\{\frac{a+d}{(a+2 R) d}\right\}^{2} V_{d r o p}(\text { for square film, } a=b) \\
& \text { where } R=\sqrt[3]{\frac{\sin ^{3} \theta}{\pi\left(-\cos \theta+\frac{1}{3} \cos ^{3} \theta+\frac{2}{3}\right)} V_{d r o p}}
\end{aligned}
$$

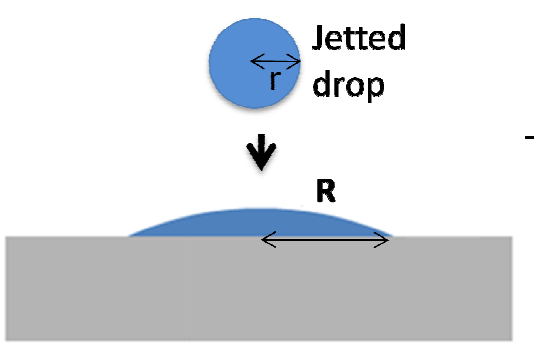

(a)

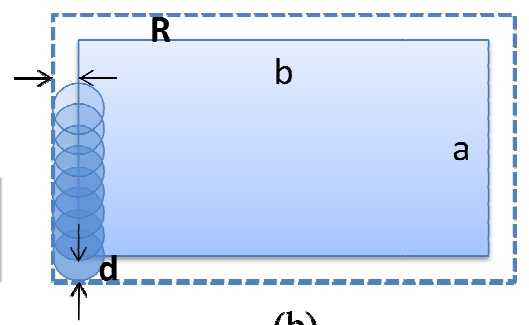

(b)

Figure 10. (a) Independent drop jetted onto a partial wetting substrate, which has a spherical cap shape with radius, R. (b) Printed rectangle with extended boundary caused by the drop radius, which has a designed size of $\mathrm{a}$ and $\mathrm{b}$ for each side.

The film thickness model including extended boundary shows a good accordance with measured data from both printer systems as shown in Figure 11. However, at smaller drop spacing the measured film thickness data for the $100 \mathrm{pL}$ drop Microfab system deviates from the model in a bulging phenomenon. The edges of a film bulge when a film is printed with smaller drop spacing. (See Figure 6.) The increased area caused by bulging eventually decreases the ink 
volume per unit area, resulting in the deviation seen in the measured data at smaller drop spacing in Figure 11. The bulging phenomenon also distorts the shape of desired patterns. In order to increase the maximum thickness beyond what can be reached due to bulging, the ink concentration can be increased.

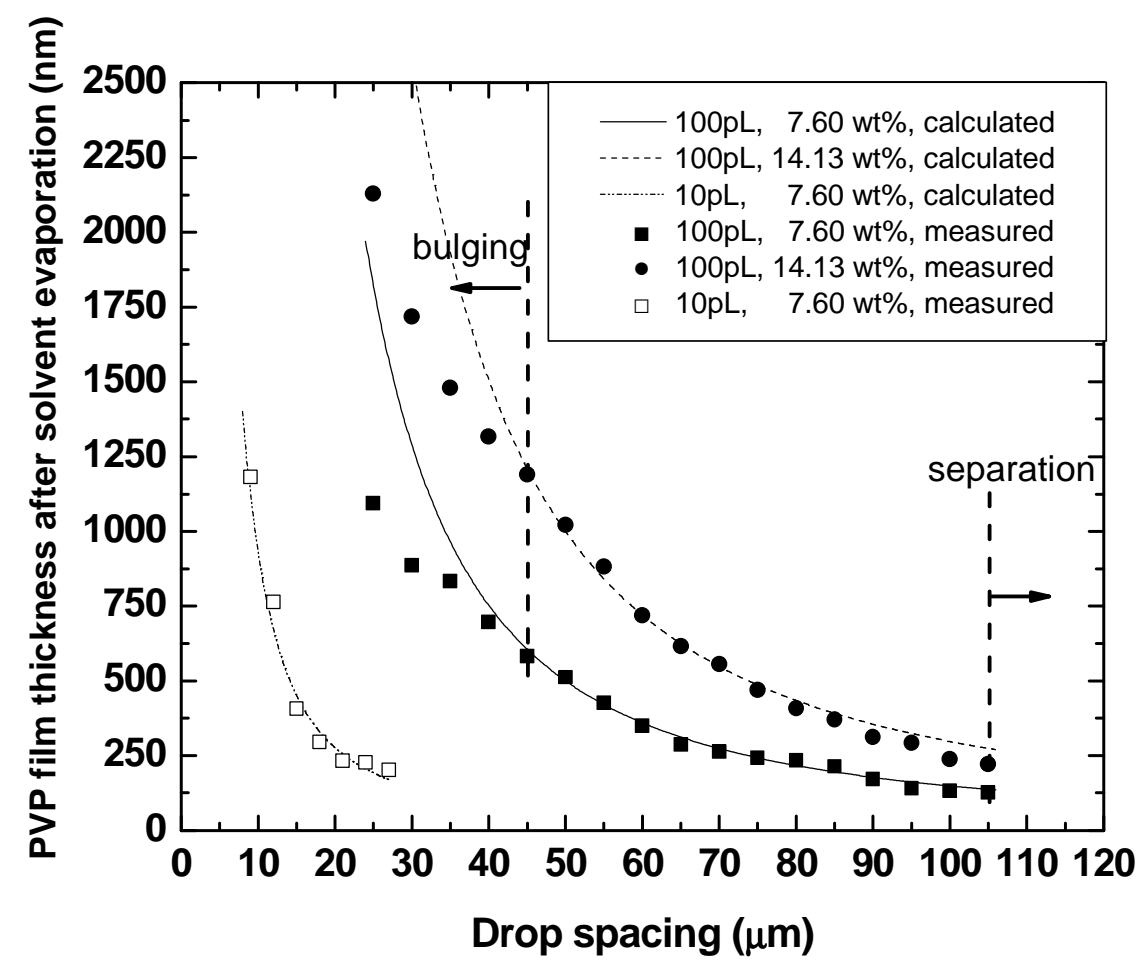

Figure 11. Film thickness modeling of equation (S2) and measurement data for $500 \mu \mathrm{m}$ square PVP films printed by the Microfab printer (115.5 $\mu \mathrm{m}$ diameter of a circular cap on Corning 1737) and $200 \mu \mathrm{m}$ by the Dimatix printer ( $45 \mu \mathrm{m}$ diameter).

\section{B. Bulging of Printed Film beyond Advancing Contact Angle}

First, we develop a simple hydrostatic model for the width of merged lines to understand the bulging phenomena quantitatively. By comparing the contact angle at the edge of merged lines with advancing contact angle, we can determine whether a bead bulges or not. 


\section{a. Two Lines Impinging}

The cross-section of a single line can be assumed as a cylindrical cap (The bond number for our lines is $2 \cdot 10^{-3}$ ), darker area A1 in Figure 12. The area A1 can be calculated by subtracting A2, the triangle area, from the sector whose central angle is $2 \theta$.

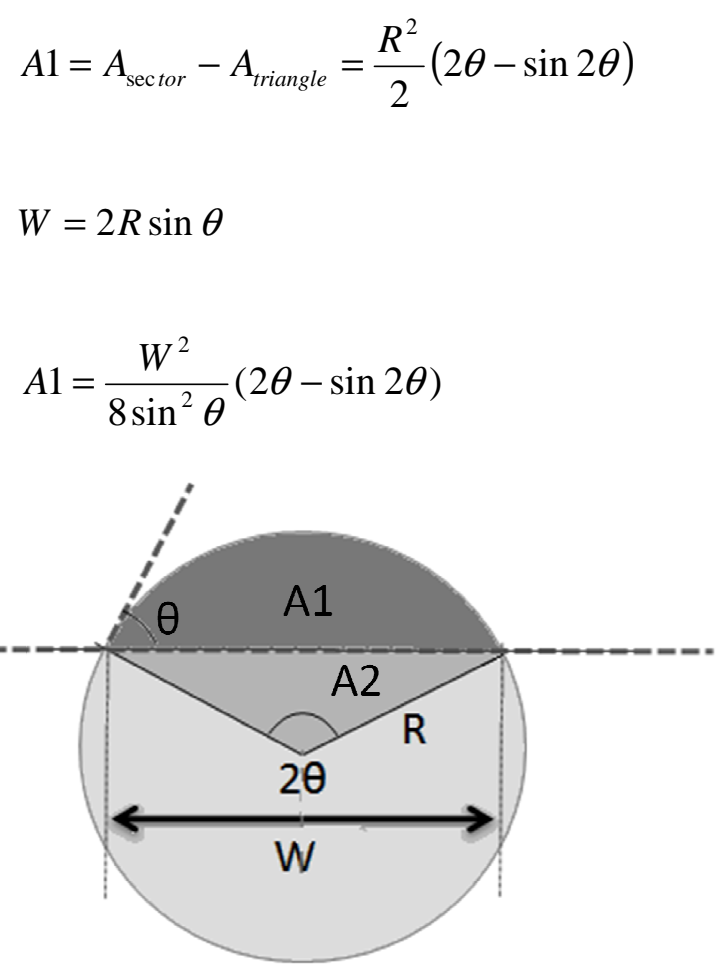

Figure 12. Circular segment geometry. The circular segment, A1, can be assumed as a crosssectional area of a single line. Therefore, $\theta$ is equilibrium contact angle and $W$ is the width of a single line.

Thus, its width is determined only by its contact angle and the cross-section area of the cylindrical cap line. Since the cross-section area of a stable line is determined by the total volume of a bead over the line length, the area is simply expressed as (6).

$$
A 1=\frac{(\text { numberof drops }) \times V_{\text {drop }}}{(\text { numberof } d r o p s) \times d}=\frac{V_{d r o p}}{d}
$$


By combining (5) and (6), the width of a single cylindrical cap line is given as (7).

$$
W_{\text {sin } g l e}=\sqrt{\frac{8 \sin ^{2} \theta V_{d r o p}}{(2 \theta-\sin 2 \theta) d}}=K \sqrt{\frac{V_{d r o p}}{d}}
$$

where $K$ is equal to 3.59 for the advancing contact angle of $7.60 \mathrm{wt} \%$ PVP ink, $26.0^{\circ}$.

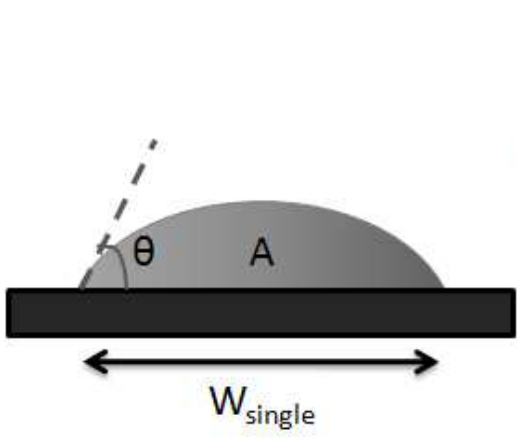

Printing direction (1) Bottom to top (2) Left to right

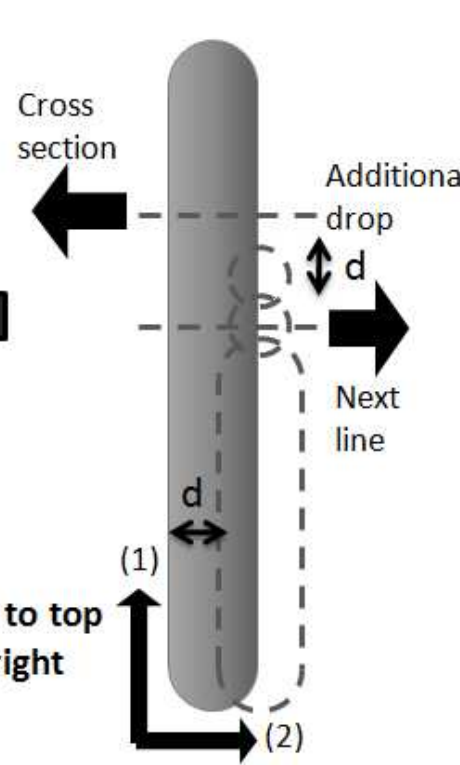

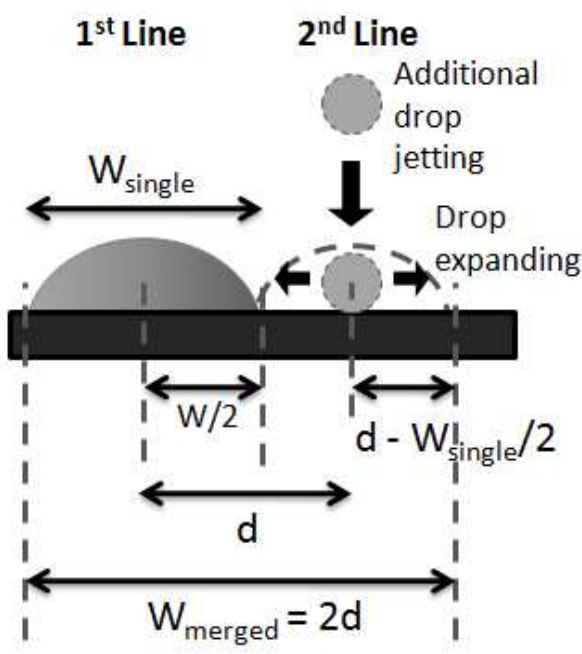

(c) cross-sectional view as second line is printed
(b) cylindrical cap lines and additional jetted drop cross-sectional view
(a) single line

\section{View}

Figure 13. Geometry of printed film; (a) single line cross-sectional view (b) cylindrical cap lines and additional jetted drop (c) cross-sectional view as second line is printed.

After the first line is printed with drop spacing, $d$, from bottom to top as in Figure 13(b), the second line is printed next to the first line with spacing, $d$, to the right. The cross-sectional view when the second line is printed is shown in Figure 13(c). When the spacing between two lines is less than the width of a single line, the second line touches the first printed line, and the two lines merge into a single bead (we ignore evaporation of solvent between printing of successive lines 
in this analysis; for typical printing speeds and solvent evaporation rates, this is a good assumption for successively printed lines in our experiment). At that point, the width of two merged lines is the sum of width of the first line and the distance over which the second line spreads as shown in Figure 13(c) and (8).

$$
W_{\text {merged }}=W_{\text {sin } g l e}+2\left(d-\frac{W_{\text {sin } g l e}}{2}\right)=2 d
$$

After the two lines merge, any further spreading is determined by the advancing contact angle for the new bead. We now endeavor to compare the widths of a just-merged bead with that of the same bead spread to its equilibrium width. Substituting twice the ink volume into equation (7) to represent two lines worth of printed drops, we find that the width of the new bead is simply $\sqrt{2}$ $W_{\text {single }}$.

If the merged line width, found with equation (8), is smaller than the line width maintaining the advancing contact angle, $\sqrt{2} W_{\text {single }}$ for two lines, then the contact angle on each side of the merged bead is larger than the advancing contact angle. Consequently the bead will flow until its contact angle is reduced to the advancing contact angle. This outflow is the bulging phenomenon seen in Figure 6.

\section{b. Generalization to Many Printed Lines}

This same approach can be extended to multiple lines as well. Bulging continues whenever a new line adds fluid such that the new, merged bead would exceed its advancing contact angle. Replacing two lines worth of ink in the case of two lines impinging with $n$, the number of printed lines, it is possible to use the same method to determine the bulging growth when $\mathrm{n}^{\text {th }}$ line is 
added, leading to the following inequality (9) for the condition where the contact angle of the merged bead is less than or equal to the advancing contact angle.

$$
\frac{d}{W_{\text {sin gle }}} \geq \frac{1}{\sqrt{n}}
$$

When the inequality (9) is satisfied, the printed bead stops bulging. Therefore, the number of lines that have to be printed to stop bulging for a certain constant drop spacing can be calculated and is shown in Figure 14(b). To test the model, ten lines are sequentially printed from the left to the right. The boundary of each dried feature is extracted, aligned, and overlaid in Figure 14(a). Since only the left hand side bulging extends beyond the feature's footprint, we look there to view the bulge's growth. At smaller drop spacing, more lines are printed before the film contact angle falls beneath the advancing contact angle. Measured data show a good fit when the drop spacing is large, but it deviates from the model at smaller drop spacing, predicting more lines than actually are actually observed to grow a bulge. This error is likely due to evaporation losses, which have not been accounted for in the above analysis. Because it takes more time to print the same length of line with smaller drop spacing, more evaporation occurs, removing ink that would otherwise contribute to the bulge.

As shown in Figure 14(a), the bulging always begins from the bottom of each line. As Duineveld explained the bulging phenomenon in his work, the bulge occurs near the starting point of printing due to the transition from spherical to cylindrical shape. Because the end of a line where drops are added has higher pressure due to extra curvature there, the added liquid flows toward the initial bulge where the pressure is lower, and the initial bulge is enlarged instead of equalizing the width of a line. 

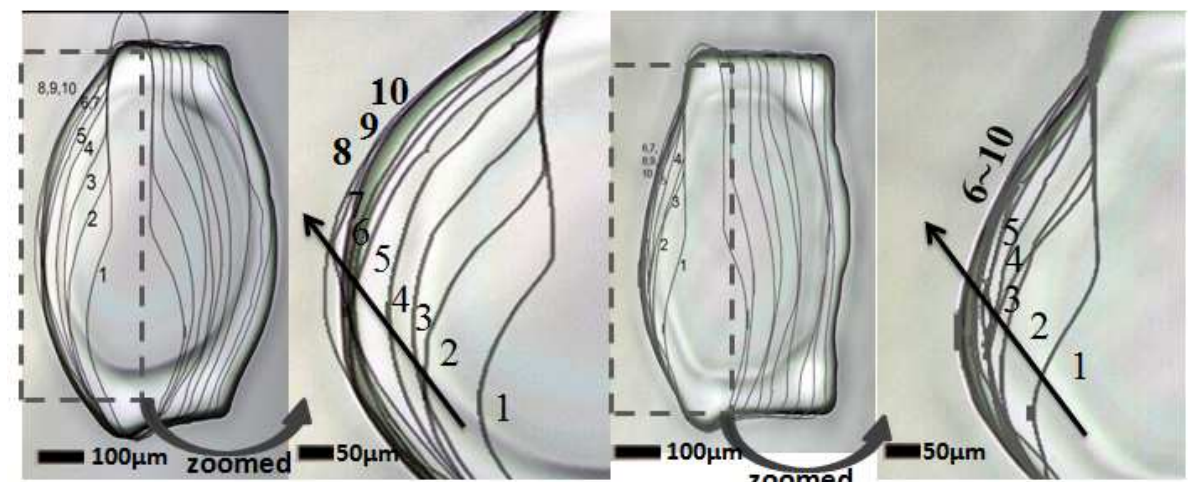

Drop spacing: $\quad 30 \mu \mathrm{m}$

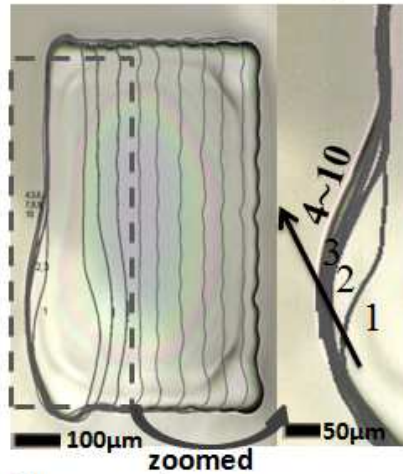

Drop spacing:
$60 \mu \mathrm{m}$
$40 \mu \mathrm{m}$

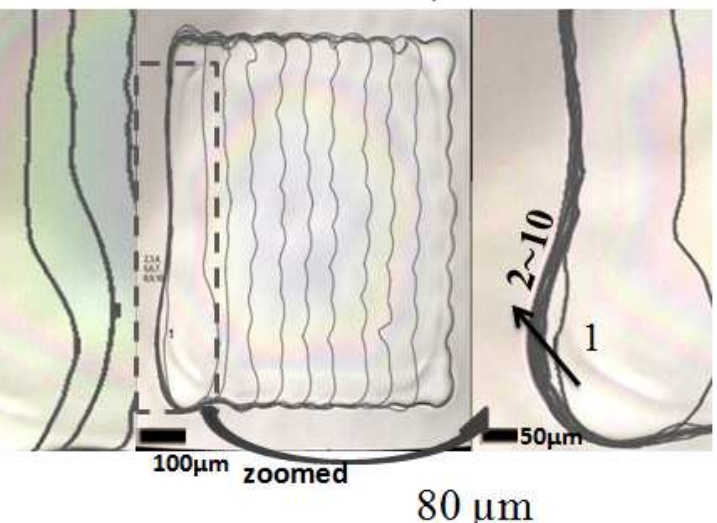

(a)

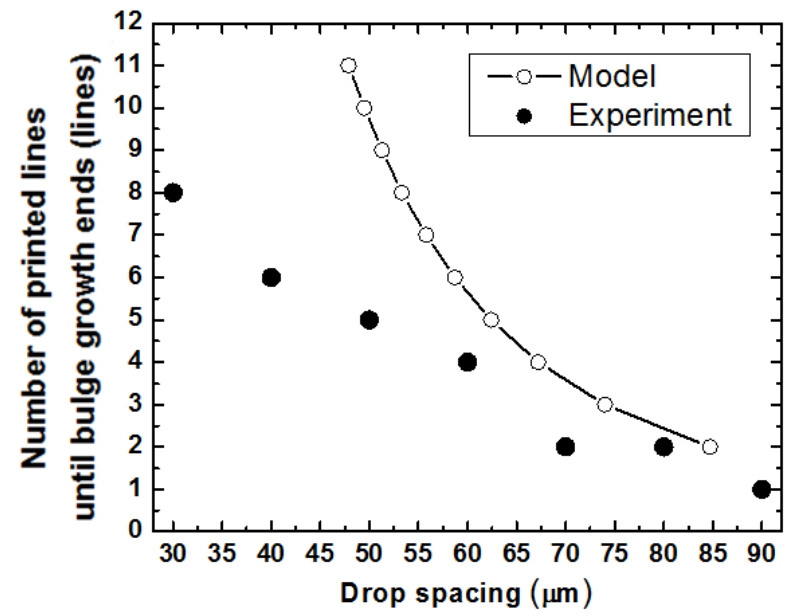

(b)

Figure 14. (a) Overlays of one to ten sequentially printed $750 \mu \mathrm{m} 7.60 \mathrm{wt} \%$ PVP lines at noted drop spacing on Corning 1737 (100 pL drop volume). Left edge is zoomed 1.5 times horizontally and 3 times vertically from the left full image of beads. The boundary of each step is extracted and overlapped with first printed line. (b) The number of lines until bulge growth ends at different drop spacing for our hydrostatic model and experiment data in (a) 


\section{Separation of Printed Film below Receding Contact Angle}

\section{a. Film Separation Model}

The film breakup phenomenon seen in Figure 7 can also be explained by the 1D simple hydrostatic model developed in the previous section. When the equality in (9) is satisfied, the merged bead has the contact angle which defines $W_{\text {single }}$. Based on the 1D hydrostatic model, the width of merged lines which have a contact angle, $\theta$, on each side is determined by the contact angle, drop spacing, drop volume and number of printed lines as in equation (10).

$$
\begin{aligned}
W_{\text {equilibrium }} & =f(d, n, \theta) \\
& =K \sqrt{\frac{V_{d r o p}}{d} \sqrt{n}} \\
& =\sqrt{n} W_{\text {sin gle }}
\end{aligned}
$$

As shown in Figure 13(c) and (8), the width of merged bead is $n d$. If $W_{\text {equilibrium }}$ in (10) is the same as $n d$, then the merged bead can stay at the contact angle in (10) and does not require spreading to meet the hydrostatic equilibrium. From this relationship, the width of a bead which has a certain contact angle on each side for certain drop spacing can be obtained as (12).

$$
\begin{aligned}
& W_{\text {equilibrium }}=W_{\text {merged }} \\
& \sqrt{n} W_{\text {sin } g l e}=n d \\
& K \sqrt{V_{\text {drop }}} \sqrt{\frac{n}{d}}=n d \\
& \frac{K \sqrt{V_{d r o p}}}{d^{\frac{3}{2}}}=\sqrt{n} \\
& W_{\text {equilibrium }}=\frac{K \sqrt{V_{\text {drop }}}}{\sqrt{d}}(\sqrt{n})=\frac{K \sqrt{V_{\text {drop }}}}{\sqrt{d}}\left(\frac{K \sqrt{V_{\text {drop }}}}{d^{\frac{3}{2}}}\right)=\frac{K^{2} V_{\text {drop }}}{d^{2}}
\end{aligned}
$$


where $K$ is equal to 3.59 for $\theta_{\text {advancing }}$ of $7.60 \mathrm{wt} \%$ PVP ink, $26.0^{\circ}$ and $K$ is equal to 4.87 for $\theta_{\text {receding }}, 14.4^{\circ}$. By using the equation (12), it is possible to set up a framework as shown in Figure 15 to anticipate the contact angle of a printed bead of a given width, printed at a given drop spacing. Based on the established boundaries for bulging, proper shape definition, and bead separation, we can then predict the specific regime within which a pattern will be printed.

Our 1D hydrostatic model has an assumption of infinite length of the bead. Therefore, the model has a limitation to be accurately applied to 2 dimensional square films. However, the framework in Figure 15 is useful to roughly anticipate the behavior of printed square films since the square films consist of merged lines. As shown in Figure 15, printed square films fit well into the boundaries defined by our 1D hydrostatic model. If drop spacing and film size is below the line defined by equation (12) with advancing contact angle $(K=3.59)$, films mostly show bulging behavior. If a film is located above the line defined by equation (12) with receding contact angle $(K=4.87)$, the printed film either dewets or breaks up into smaller beads. Printed films located between those two lines tend to retain a stable square shape. There is a tendency for films to be separated at smaller size or smaller drop spacing than critical values of size or drop spacing. This is likely caused by two issues. Firstly, the 1D model cannot accurately predict the contact angle of square films, and secondly the total volume of the films is reduced due to evaporation of solvent as films are printed. However, despite these errors, we in all cases we clearly see that hydrostatic concerns prove to define the experimental space within which uniform films can be printed. 


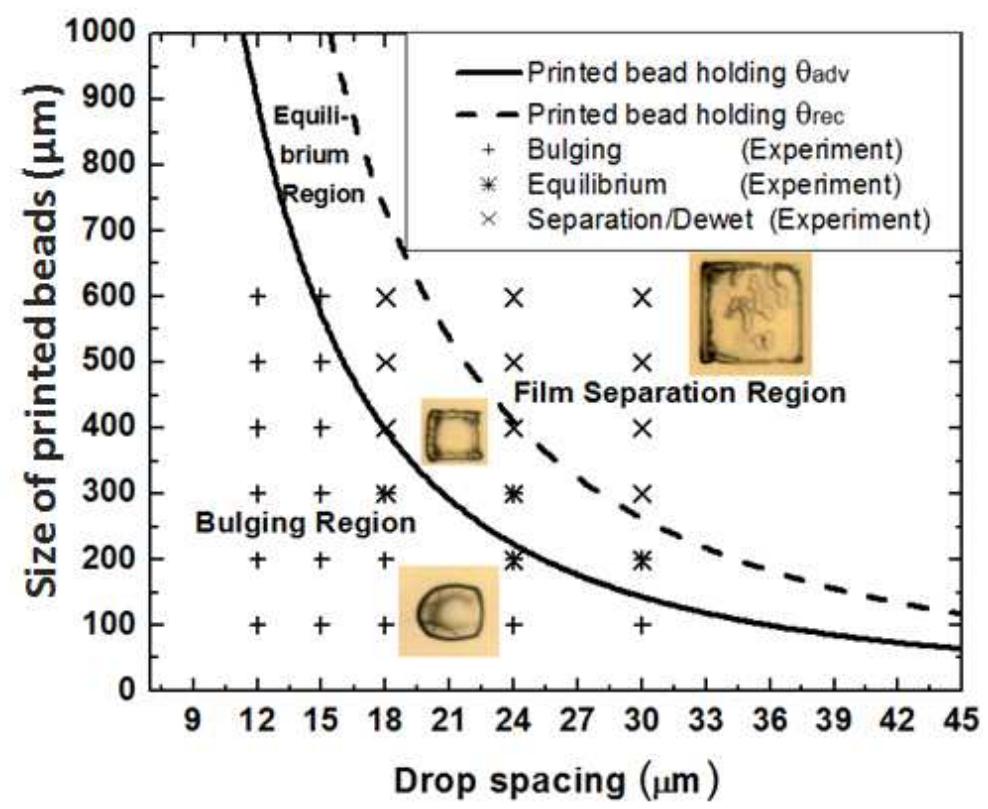

(a)

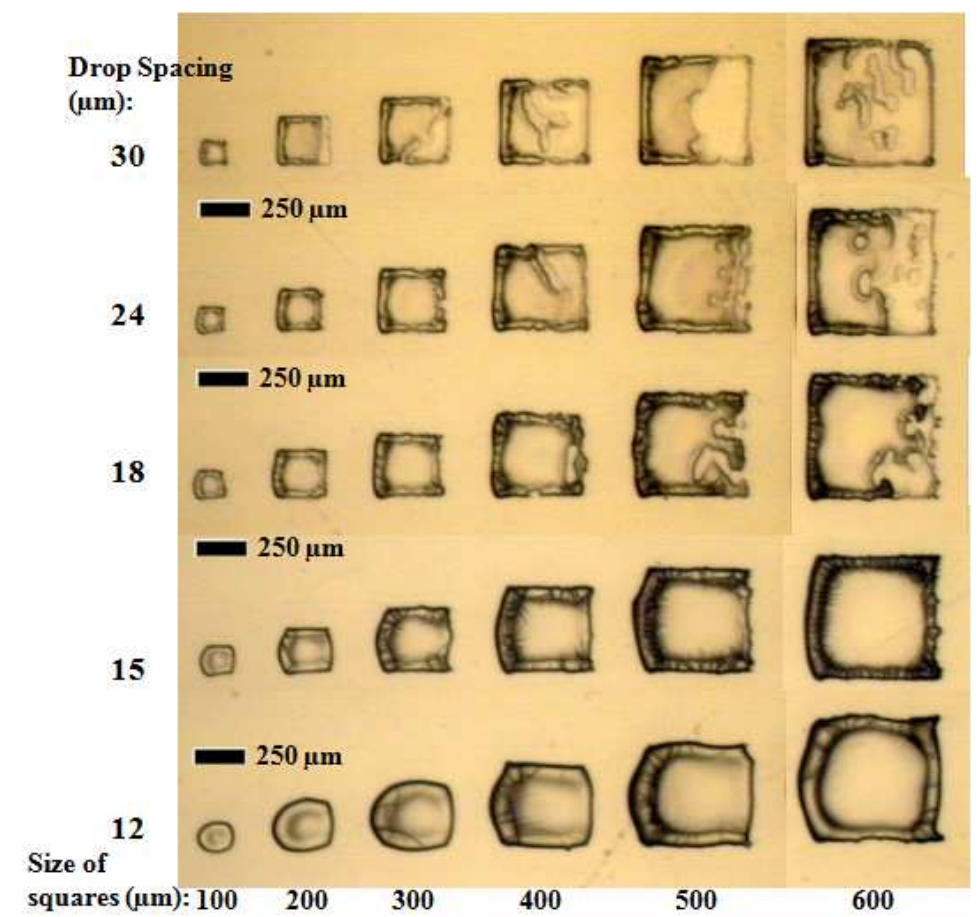

(b)

Figure 15. (a) Printed film shape determination based on the size and drop spacing of the film (b) $7.60 \mathrm{wt} \%$ PVP PVP films with different size and drop spacing (10 pL drop volume, single nozzle used, on Corning 1737) 


\section{b. Multiple Nozzle Printing}

Although much inkjet printing research has been done with single nozzle systems, multiple nozzle printing is desirable for higher throughput. Even if single nozzle printing results show good fitting to the model, unexpected characteristics such as asymmetric edge shape and earlier film-separation tendency have been observed due to unconsidered evaporation in the model. When lines are printed with shorter time intervals or at the same time using multiple nozzles, it is acceptable to ignore evaporation of ink during printing and evaporation-induced pinning.

In A-1 of Figure 16,the left edge is observed tobulge, but the right edge slightly dewets the surface, showing asymmetry. However, when six nozzles are used to print the same film in A-2 of Figure 16, bulging is observed on both sides, and therefore the pattern becomes more symmetrical. Based on the hydrostatic condition, the film is also within the bulging region. Since it takes a smaller amount of time to form the pattern when performing multiple nozzle printing, evaporation is suppressed during printing and thus, there is less change of total volume. As shown in B-1,2 of Figure 16, multiple-nozzle printed films dewet slightly, but do not break up; a single-nozzle printed film of the same size with the same drop spacing is separated, however. However, due to quicker printing, the amount of time taken for forming evaporation-induced pinned line edges is also decreased, resulting in a more surface-energy minimized round shape. This is more clearly observed in (C-1 and 2) of Figure 16. In C-1, a single nozzle allows enough time for printed lines to be dried and pinned, but the last portion of the printed volume is separated for surface energy minimization. Therefore, the square film still maintains its square boundary. However, in C-2, six-nozzle-printing jets the total volume of ink before the square boundary is formed and the boundary lines recede to minimize the surface energy. These thus form more clearly separated smaller films. 
Overall, we see that the same hydrostatic conditions should be also considered in printing films with multiple nozzles; considering these, we note that multiple-nozzle printed films have symmetry in their shape and tend to fit the simple hydrostatic model slightly better due to slower evaporation relative to print speed. The tendencies discussed are seen in Figure $\mathbf{1 7}$ as well.

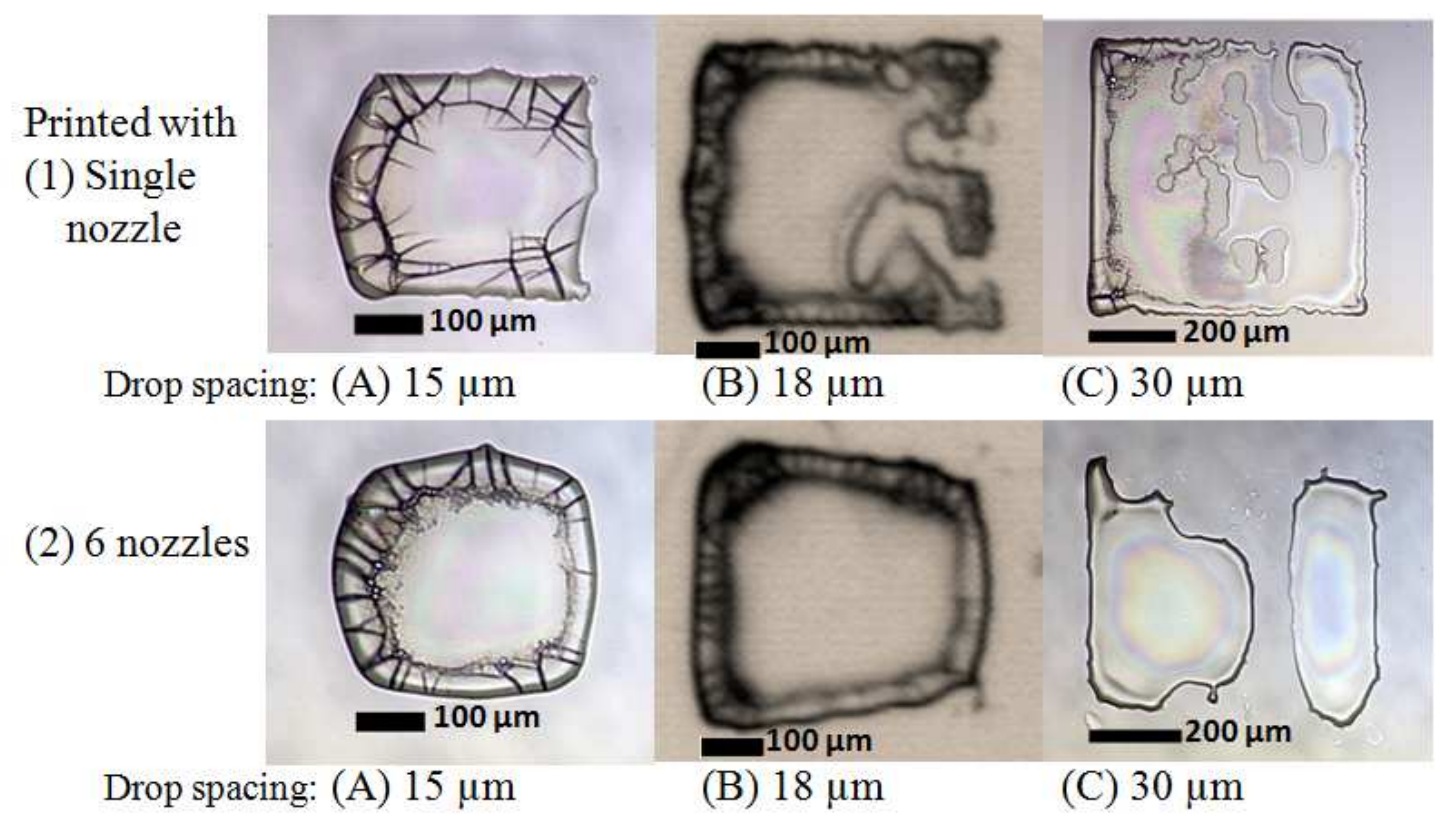

Figure 16. Identical films printed with different numbers of nozzles used. (A) $300 \mu \mathrm{m}$ square is printed with $15 \mu \mathrm{m}$ drop spacing, (B) $500 \mu \mathrm{m}$ square with $18 \mu \mathrm{m}$ drop spacing and (C) $600 \mu \mathrm{m}$ square with $30 \mu \mathrm{m}$ drop spacing both by single nozzle (1) and six nozzles (2). 


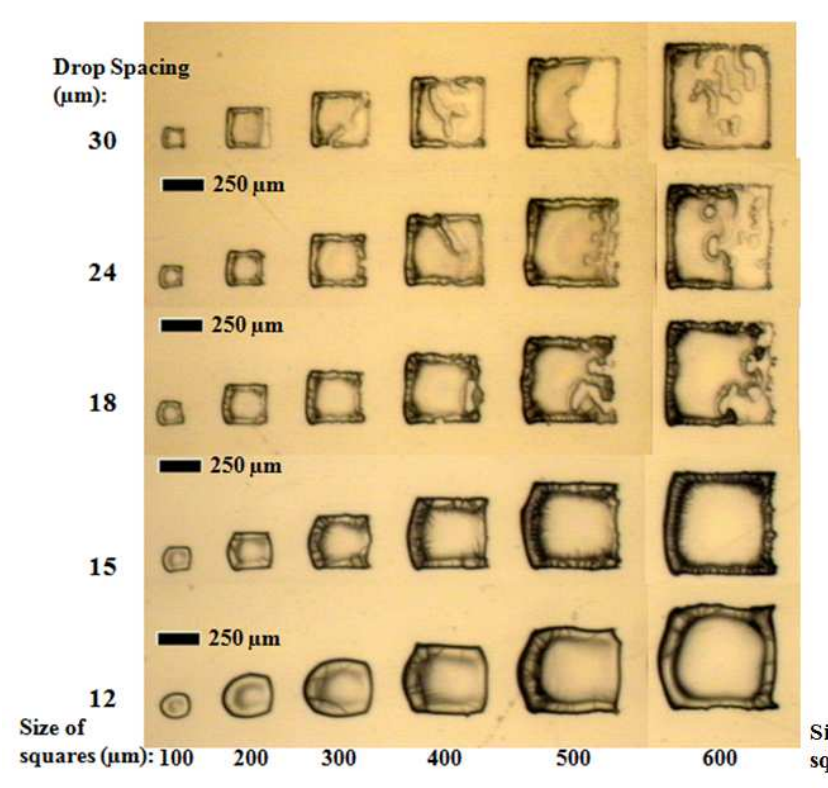

(a) Printed with Single Nozzle

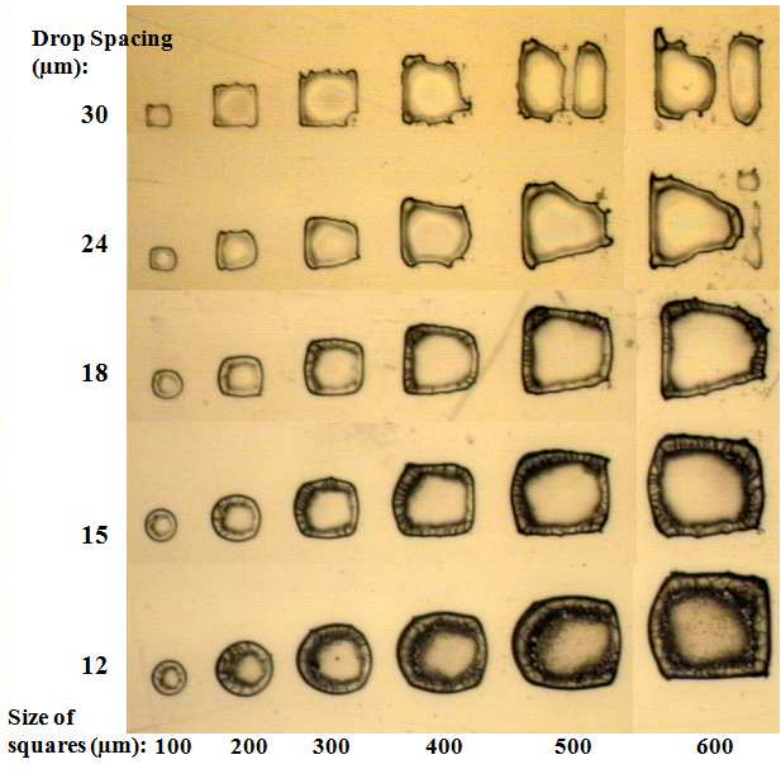

(b) Printed with 6 Nozzles

Figure 17. Identical films printed with different numbers of nozzles used; (A) printed with single nozzle (B) printed with 6 nozzles. 


\section{Conclusion}

In this work, we have provided a simple analytic framework for the optimization of printing of two dimensional patterns using droplet-on-demand printing. We showed a simple film thickness model with a good fit to our printed polymer films. We have also demonstrated and explained limitations of the model caused by bulging or not merged drops. In order to increase the maximum thickness beyond what can be reached due to bulging, the ink concentration can be increased. This model enabled us to be able to accurately predict the thickness of printed films. To develop an understanding of bulging and separation of a film, we suggested a onedimensional hydrostatic model. A large amount of ink in a given bead, such that its contact angle is higher than the advancing contact angle, causes the bead to advance and wet the substrate. If the amount of ink is not enough to meet receding contact angle, the film breaks up into smaller films to minimize its surface energy. Therefore, contact angle hysteresis has to be considered carefully for a complete and uniform printed film.

Although the simple 1D hydrostatic model in this work helps provide an understanding of the behavior of printed films and proves its usefulness, the model is applicable accurately only when the patterns are quasi-1D structures; errors in the 1D approximations manifest themselves as the shape deviates from this regime. When both sides of a pattern become comparable, it is necessary that the entire contact angle around the pattern has to be considered so that the bulging and dewetting can be anticipated not only at two edges but around the boundary; this is beyond the bounds of this report. What this model clearly does establish, however, is the limitations imposed by hydrostatic considerations on films formed using constantly-spaced droplets. Fundamentally, there are finite size regimes for given drop spacing and contact angle conditions 
over which well-defined patterns without bulging or bead breakup can be achieved. New methods have to be developed to print larger patterns on a substrate with unchangeable contact angle hysteresis. Electronic devices such as TFTs, OLEDs and solar cells have multilayer structures. Modifying the surface energy of each layer is not always possible because chemical or physical treatments cause the degradation of the device characteristics such as carrier recombination rate, carrier transport and electric field endurance. Thus, when the surface energy is inflexible, this model dictates with good accuracy that is not possible to print larger features by constant drop spacing printing due to the receding contact angle. This therefore places a specified and predictable limitation on pattern formation conditions for printed structures. 


\section{References}

[1] Duineveld, P. C. J. Fluid Mech., 2003, 477, 175-200.

[2] Soltman, D.; Subramanian, V. Langmuir, 2008, 24, 2224-2231

[3] Ozawa, K.; Nishitani, E.; Doi, M. Jpn. J. Appl. Phys., 2005, 44, 4229-4234.

[4] Li, Y.; Fu, C.; Xu, J. Jpn. J. Appl. Phys., 2007, 46, 6807-6810.

[5] Kaneda, M.; Ishizuka, H.; Sakai, Y.; Fukai, J. AlChE J., 2007, 53, 1100-1108.

[6] Tekin, E.; de Gans, B.; Schubert, U. J. Mater. Chem., 2004, 14, 2627-2632.

[7] Klauk, H.; Halik, M.; Zschieschang, U.; Schmid, G.; Radlik, W., J., Appl. Phys., 2002, 92, 5259-5263.

[8] Yoon, M.; Yan, H.; Facchetti, A.; Marks, T., J. Am. Chem. Soc., 2005, 127, 10388-10395.

[9] Deegan, R.; Bakajin, O.; Dupont, T.; Huber, G.; Nagel, S.; Witten, T., Nature, 1997, 389, 827-829.

[10] Kim, D.; Jeong, S.; Park, K.; Moon, J., Appl. Phys. Lett., 2006, 89, 264101-3.

[11] Tseng, H.; Subramanian, V., IEDM, 2009, 15, 391-394. 


\section{APPENDIX}

\section{Puddle Thickness Limited by Gravity}

Gravity flattens partially wetting films to height given below. ${ }^{1}$

$$
e=2 \sqrt{\frac{\gamma}{\rho g}} \sin \left(\frac{\theta_{E}}{2}\right)
$$

where $e$ is the puddle thickness, $\gamma$ is the surface tension, $\rho$ is the density of liquid and $\theta_{E}$ is the equilibrium contact angle. With the parameters of 1 -hexanol, ${ }^{2}$ the thickness in (S1) can be calculated to be about $800 \mu \mathrm{m}$. The height of printed PVP films before evaporation is from 10 $\mu \mathrm{m}$ to $150 \mu \mathrm{m}$, and much smaller than the thickness calculated from (S1). Therefore, we can ignore gravitational effects when calculating shapes of printed films in this work.

\section{REFERENCES FOR APPENDIX}

[1] de Gennes, P.; Brochard-Wyart, F.; Quéré, D., Capillarity and Wetting Phenomena-Drops, Bubbles, Pearls, Waves; Springer, 2004; Chapter 2.

[2] Piñeiro, M.; García, J.; de Cominges, B.; Vijande, J.; Valencia, J.; Legido, J., Fluid Phase Equilibria, 2006, 245, 32-36. 